\title{
Visuo-spatial processing and dimensions of schizotypy: figure- ground segregation as a function of psychotic-like features ${ }^{1}$
}

\author{
Elias Tsakanikos and Phil Reed
}

\begin{abstract}
The aim of the reported study was to determine whether the ability to segregate a simple figure embedded in a complex visual ground, was associated with psychotic-like features in a sample of undergraduate students. The participants ( $N=100)$ were tested on the Hidden Figures Test, as well as the Raven's Progressive Matrices, and completed a multi-dimensional schizotypy inventory (O-LIFE). The IQ scores were positively related to the number of correct responses on the Hidden Figures Test, but were unrelated to any of the schizotypy measures. Impaired Performance on the Hidden Figures Test was associated with negative schizotypy ('Introvertive Anhedonia'), and enhanced performance was associated with the 'Impulsive Non-Conformity' scale. Performance on the Hidden Figures Test was independent of the positive ('Unusual Experiences'), and the disorganized ('Cognitive Disorganization'), schizotypy. The results are discussed in terms of a putative involvement of the frontal lobes in the negative symptomatology of schizophrenia, and in top-down (goal-driven) perceptual processing, as well as the possible compensatory functional aspect of impulsivity in terms of allocating attention.
\end{abstract}

Keywords: field dependence/independence; figure-ground perception; flexibility of closure; frontal lobes; general fluid intelligence; impulsivity; negative schizotypy; schizophrenia

Schizophrenia has long been associated with various deficits of attention (see Braff, 1993; Nestor \& O’Donell, 1998, for reviews). A variety of evidence suggests that schizophrenic patients are relatively impaired in their ability to ignore irrelevant stimuli, as assessed by latent inhibition (Baruch, Hemsley \& Gray, 1988; Gray, Pilowsky, Gray, \& Kerwin, 1995; Lubow, Kaplan, Abramovich, Rudnick \& Laor, 2000). The same pattern of results also has been reported for healthy individuals that score high on schizotypy measures (Lipp \& Vaitl, 1992; Lubow, Ingberg-Sachs, Zahlstein-Orda, \& Gewirtz, 1992; Tsakanikos \& Reed, in press). Additionally, it has been shown that saccadic latency in the presence of irrelevant stimuli is prolonged to a greater extent in schizophrenic than it is in non-schizophrenic patients (Schwartz \&

\footnotetext{
${ }^{1}$ Author's note. This is a pre-print version of a paper which was subsequently published as a journal article (final version): Tsakanikos, E., \& Reed, P. (2003). Visuo-spatial processing and dimensions of schizotypy: figure-ground segregation as a function of psychotic-like features. Personality and Individual Differences, 35, 703-712.
} 
Evans, 1999), suggesting a deficient disengagement of attention from the irrelevant stimulus in schizophrenia.

Attentional deficits in schizophrenia have been attributed to an inadequate topdown (knowledge-driven) perceptual processing, as compared to the bottom-up (stimulus-driven) processing (Van den Bosch, 1995; Nestor \& O’Donell, 1998; but see Jensterle, Mlakar, Vodusek \& Frith, 2000). It has been shown, for example, that schizophrenics cannot utilize top-down (contextual cues) factors to segregate relevant from irrelevant acoustic material presented in the auditory stream, but they can use sufficiently bottom-up (acoustic properties) factors (Silverstein, Matteson \& Knight, 1996). Similarly, top-down, experience-based influences are limited when schizophrenic patients process unstructured visual patterns (Silverstein, Bakshi, Chapman \& Nowlis, 1998).

Top-down influences seem to modulate figure-ground segregation in visual processing under conditions of perceptual ambiguity (Humphreys \& Mueller, 2000; Vecera \& O’ Reilly, 1998). Poor performance in figure-ground segregation has been demonstrated in schizophrenic patients (Eimon, Eimon \& Cermak, 1983), and this has been associated with the presence of delusions and hallucinations (Liddle, 1988) suggesting poor top-down processing, a finding that is consistent with the literature above. Nevertheless, general perceptual organization in terms of utilizing the gestalt principles has been found to be intact in schizophrenia (Chey \& Holzman, 1997; Knight, Manoach \& Hershenson, 2000).

A traditional visuo-spatial test that involves figure-ground segregation is the Hidden Figures Test (Ekstrom, French, Harman \& Dermen, 1976). This test requires the observers to identify which one of five simple figures (perceptually present) is hidden in a complex visual configuration. Although an impaired performance in the Hidden Figures Test has been reported in schizophrenic patients (Neville, 1995), there has been no effort to relate this deficit to the symptomatology of the disorder.

A frequent problem in schizophrenia research is the interpretational limitations that arise in terms of the reported neuro-cognitive deficits. It is not always clear whether the identified deficits are due to medication, poor motivation, disruptive effects of active psychotic symptoms, or the psychotic syndrome per se. Therefore, clinical research is often complemented through assessing healthy, psychometrically identified, high schizotypy scorers. Importantly, the approach of testing individuals that show some sub-clinical features of schizophrenia facilitates experimentation on schizophrenia-related hypotheses by making feasible the introduction of more complex and demanding experimental procedures that might not have been applicable to a clinical population.

The identification of psychotic-like features in the general population has often taken the form of a multi-dimensional approach, following various factor-analytic studies that have revealed three or four factors underlying the construct of schizotypy (Bentall, Claridge \& Slade, 1989; Vollema \& Van den Bosch, 1995). The OxfordLiverpool Inventory of Feelings and Experiences (O-LIFE; Mason, Claridge \& Jackson, 1995), for example, assesses four dimensions of schizotypy. The first three dimensions phenomenologically correspond to a three-factor model of schizophrenia (Liddle, 1987): a positive ('Unusual Experiences'), a negative ('Introvertive Anhedonia'), and a disorganized factor ('Cognitive Disorganization'). The fourth dimension ('Impulsivity Nonconformity') refers to the impulsive, aggressive and asocial aspects of psychosis, and is largely based on Eysenck's Psychoticism scale (Eysenck \& Eysenck, 1975). Contributing to the experimental validity of this schizotypy inventory, various studies have confirmed that high schizotypy scorers, as 
identified by the O-LIFE sub-scales, demonstrate the same neuro-cognitive deficits as the schizophrenic patients (e.g., Burch, Steel \& Hemsley, 1998; Goodarzi, Wykes \& Hemsley, 2000; Rawlings \& Goldberg, 2001).

The purpose of this investigation was to assess figure-ground segregation by employing the Hidden Figures Test, and replicate in hypothetical high schizotypy scorers, as identified by the O-LIFE inventory, the finding that figure-ground segregation is impaired in schizophrenia (Eimon et al., 1983, Liddle, 1988, Neville, 1995). Furthermore, it was sought to explore further the relationship between psychotic-like features and Hidden Figures Test within a multi-dimensional schizotypy approach. Given the putative link between this test and the frontal lobe functioning (Della Salla, Gray, Spinnler \& Trivelli, 1998; Federico, 1984), which is itself putatively associated with the negative psychotic symptoms (e.g., Ananth, Burgoyne, Gadasalli, Aquino, 2001; Lahti, Holcomb, Medoff, Weiler, Tamminga, Carpenter, 2001; Schmajuk, 2001), it was expected that performance in the Hidden Figure Test would be particularly related to the negative psychotic-like features identified psychometrically.

An extensive review of various studies (see McKenna, 1984) suggests a close relationship between the ability to identify a simple figure embedded in a complex configuration and general intelligence, as assessed by the Raven's Progressive Matrices. Given the often-reported intellectual decline associated with schizophrenia (Bilder, Lipschutz-Broch, Reiter \& Geisler, 1992; Gold, Arndt, Nopoulos, O’Leary \& Andreasen, 1999), the Raven's Progressive Matrices were administered in the present investigation in order to control for the IQ level of the participants.

\section{Method}

\section{Participants}

One hundred undergraduate psychology students (22 males and 78 females) took part in this study as a part of a course requirement. Their age ranged from 18 to $53(\mathrm{M}=19.6, \mathrm{SD}=4.5)$. The participants were naïve to the purpose of the investigation.

\section{Cognitive Tasks}

Hidden Figures Test. The Hidden Figures Test (Ekstrom et al., 1976) has been often used as a measure of field-dependence/independence (e.g. Noppe, 1985; Schmidt \& Stephans, 1991), and as a task assessing frontal lobe function in neuropsychological patients (e.g., Della Salla et al., 1998). In this test, each participant is presented with five simple figures (available throughout the task) and thirty-two complex figures/problems. Participants identify which one of the five simple figures is hidden in each of the complex figures. The test is divided in two sections, each of which consists of sixteen problems, and the level of difficulty remains the same within and across the sections.

The time available to complete the task was twenty minutes. The number of correctly answered problems was the dependent measure of this task. Incorrect responses were recorded as well to control for the false alarm rate.

Raven's Progressive Matrices. The Raven's Standard Progressive Matrices (Raven, Court, \& Raven, 1977) have been argued to provide a non-verbal, culturally free measure of the general ability to solve novel problems and adapt to new situations (fluid intelligence). This IQ test has been shown to have acceptable validity and reliability (Carroll, 1993; Lezak, 1995). The test consists of sixty items, grouped 
into five sets of twelve (A to E), and the level of difficulty gradually increases across sets. The time available to complete the task was forty-five minutes. The dependent measure was the total number of correctly answered items.

\section{Schizotypy}

The Oxford-Liverpool Inventory for Feelings and Experiences (O-LIFE) consists of 159 items selected on the basis of factor-analytic studies of scales that have been employed in the past to assess psychotic-like features in the general population (Bentall et al., 1989; Mason et al., 1995). More specifically, it assesses the following dimensions:

'Unusual Experiences' reflects the positive symptoms of psychosis, and consists of items assessing magical thinking, unusual perceptual aberrations, and hallucinatory experiences (e.g., "When in the dark do you often see shapes and forms even though there is nothing there?"; "Are your thoughts sometimes so strong that you can almost hear them?”).

'Cognitive Disorganization' reflects the disorganized aspect of psychosis, and consists of items assessing difficulties with concentration and decision making, as well as social anxiety (e.g., "No matter how hard you try to concentrate do unrelated thoughts always creep into your mind?”; “Are you sometimes so nervous that you are blocked?”).

'Introvertive Anhedonia' reflects the negative aspects of psychosis, and consists of items assessing the lack of enjoyment from social contact, physical activities, coupled with aversion to emotional and physical intimacy (e.g., "Are you much too independent to get involved with other people?”; “Are people usually better off if they stay aloof from emotional involvements with people?”).

'Impulsive Non-conformity' consists of items assessing aggressive, anti-social and impulsive behaviour (e.g., "Were you ever greedy by helping yourself to more than your share of anything?”; “Do you ever feel the urge to break or smash things?”).

\section{Procedure}

The psychometric measures were administered in three group sessions. The instructions and the tests were administered by the authors with the help of four lab demonstrators. The participants were fully debriefed about the purpose of the study at the end of both sessions.

\section{Results}

Table 1 about here

Table 1 shows the means, standard deviations, and inter-correlations between the O-LIFE scales. The means and the standard deviations were quite similar to those reported in the original study on the development of the scales (Mason et al., 1995), as well as those reported in later studies (Rawlings \& Goldberg, 2001). In terms of the inter-correlations, the overall pattern was consistent with that obtained in the previous studies. The only exception was the absence of a significant negative correlation between 'Impulsivity Nonconformity' and 'Introvertive Anhedonia'. In 
the Mason et al. study, this was $r=-0.10, p<0.05$. Given, however, that the correlation between these two scales was low and negative in both studies, this deviation could be due to the difference in the sample size between the original study $(\mathrm{N}=508)$ and the present investigation $(\mathrm{N}=100)$.

There was a relatively low correlation $(r=0.22, p<0.05)$ between the number of correct responses on Raven's Progressive Matrices $(M=47.94, S D=5.31)$ and Hidden Figures Test $(M=11.24, S D=6.38)$, suggesting that the two measures, although related, maintained a substantial amount ( $>95 \%$ ) of non-shared variance.

Table 2 about here

To examine whether schizotypy scores can predict performance on the Hidden Figures Test, and control for the contribution of the IQ level, a multiple regression analysis was performed in two successive steps. At the first step, the number of correct responses on the Raven's Progressive Matrices was entered in the regression equation as a predictor variable. The number of correct responses on the Hidden Figures Test was the dependent variable. The overall equation was significant, $F_{(1,98)}$ $=5.01, p<0.05$, and this accounted for $4 \%$ of the total variance (adjusted $R^{2}$ ). At the second step of this analysis, the four O-LIFE scales were entered in the regression equation as predictor variables together with the number of correct responses on the Raven's Progressive Matrices. The number of correct responses on the Hidden Figures Test remained the dependent variable. The overall equation was significant, $F_{(5,94)}=6.08, p<0.001$, accounting for $20 \%$ of the total variance (adjusted $R^{2}$ ). Table 2 presents the regression coefficients for the predictor variables. The regression slopes for 'Unusual Experiences', 'Cognitive Disorganization' and 'Introvertive Anhedonia' were negative, indicating that an average increase in each of them was associated with a decrease in the dependent variable. Contrary to this pattern, the slopes for 'Impulsivity Nonconformity' and number of correct responses on the Raven's Progressive Matrices were positive. However, only 'Introvertive Anhedonia', 'Impulsivity Nonconformity' and Raven's Progressive Matrices made significant independent contributions to the dependent variable.

The number of correct responses on the Hidden Figures Test correlated negatively with the number of incorrect responses on the same task (Person's $r=$ $0.25, p<0.01)$. However, there was no other significant correlation between the number of incorrect responses and any other measures, all $p s>20$, eliminating the possibility that the observed contribution of the schizotypy measures was the result of a high false alarm rate.

\section{Discussion}

The present investigation was undertaken in order to determine whether different schizotypy dimensions could predict a top-down (knowledge/goal-driven) perceptual processing, such as segregating a simple figure embedded in a complex background, as assessed by the Hidden Figures Test. Negative schizotypy was found to be a 
significant predictor of performance on this task, in accord with past evidence that figure-ground segregation in general, and in Hidden Figures Test in particular, is impaired in schizophrenic patients (Eimon et al., 1983; Liddle, 1988; Neville, 1995). If performance on the Hidden Figures Test does reflect a frontal lobe function, as it has been argued (Della Salla et al., 1998; Federico, 1984), and negative symptoms in schizophrenia are related to a frontal lobe hypo-function (Ananth et al., 2001; Lahti et al., 2001), then the negative relationship between negative schizotypy and performance on the Hidden Figures Test could be an indication, albeit indirect, of a putative link between negative schizotypy and frontal lobe function.

There was no indication of impaired performance for people scoring high in the positive or the disorganised schizotypy, as assessed by the 'Unusual Experiences' and 'Cognitive Disorganisation' scale, respectively. Healthy participants scoring highly on each of these two scales typically demonstrate the same pattern of neuro-cognitive deficits as the schizophrenic patients, with pronounced positive or disorganized symptomatology (e.g., Burch et al., 1998; Goodarzi et al., 2000; Rawlings \& Goldberg, 2001). Therefore, the absence of an impaired performance on the Hidden Figures Test for participants who scored high on 'Unusual Experiences' and 'Cognitive Disorganisation', can reinforce further the idea that the observed impairment on the Hidden Figures Test is specific to the negative aspect of schizotypy, and possibly to negative symptomatology of schizophrenia.

The positive relationship between 'Impulsivity Nonconformity' and performance on the Hidden Figures Test was an unpredicted finding. The fact that there was no significant relationship between 'Impulsive Nonconformity' and number of incorrect responses (false-alarm rate) on the Hidden Figures Test, debilitates any explanation based on a possible tendency of high scorers on this scale to maximize their performance through employing a more "impulsive", and prone to false alarms (as a pay-off of random guessing) response pattern.

Given that the construction of the 'Impulsive Nonconformity' scale reflects antisocial and impulsive behavioural tendencies, an alternative account could be that high scorers were more likely to cheat during the group testing, than low scorers. This account, however, is limited by the absence of a relationship between 'Impulsive Nonconformity' and performance in the Raven's Progressive Matrices. The testing conditions (group administration) were the same for both Hidden Figures Test and Raven's Progressive Matrices, and there is no obvious reason to assume that high scorers on the 'Impulsive Nonconformity' scale were likely to cheat on the one test, but not on the other one.

It is possible, therefore, that elevated levels of impulsivity, as tapped by the 'Impulsive Nonconformity' scale, facilitated the detection of a simple figure hidden in a complex configuration. There is evidence that the tendency in certain individuals to act with relatively little forethought can actually be advantageous in various cognitive tasks (see, Dickman, 1993; 1996). For example, low and high impulsives differ in the degree to which attention remains fixed on a current source of input. It has been suggested (Dickman, 1993) that individuals who tend to act impulsively can shift their attention more easily from its current location than less impulsive individuals. Such differences in terms of allocating attention have been further confirmed in a visual search paradigm that involved individuals scoring high or low on impulsivity measures (Dickman, 2000), contributing further to the notion that impulsivity has both functional and dysfunctional facets. From this perspective, high scorers on the 'Impulsive Nonconformity' scale might have been facilitated from such a pattern of 
rapid attentional shifts from fixation points while searching for a hidden shape in a complex visual configuration.

General intellectual ability, as assessed by the Raven's Progressive Matrices, was found related to performance on the Hidden Figures Test. This seems to be in congruence with past findings that have shown that the ability to segregate a simple figure embedded in a complex configuration is related to fluid intelligence (McKenna, 1984). Despite the often-reported intellectual decline in people suffering from schizophrenia (Bilder et al., 1992; Gold et al., 1999) there was no relationship between intelligence and any of the schizotypy dimensions. This latter finding is compatible with the view that the intellectual decline in schizophrenia is a possible result of the disruptive effect of the psychotic symptoms, rather than being a traitmarker for schizophrenia.

Although in the present investigation performance on the Hidden Figures Test was operationalised in terms of a top-down (goal-driven) perceptual processing (segregating a simple figure embedded in a complex background), alternative interpretations regarding the cognitive processes underlying this test also need to be considered. For example, it has been recently proposed (Miyake, Witzki, \& Emerson, 2001) that performance in the Hidden Figures Test can be better understood from a working-memory perspective (i.e. maintaining temporarily a simple figure, while simultaneously scanning the complex configuration and keeping track of the possible solutions). Given that working memory has been linked to general fluid intelligence, as assessed by the Raven's Progressive Matrices, (Engle, Tuholski, Laughlin \& Conway, 1999; Kyllonen, 1996) this is also compatible with an obtained significant positive relationship between performance on the Hidden Figures Test and the Raven's Progressive Matrices in this study. Consequently, there is also the possibility that the demonstrated impairment of the Hidden Figures Test in the negative schizotypy might actually reflect an impairment of working memory in negative symptomatology of schizophrenia, rather than a putative impairment that is specific to the figure-ground segregation process. The absence, however, of a relationship between negative schizotypy and performance on the Raven's Progressive Matrices makes this account less likely.

In summary, this investigation determined that impaired performance on the Hidden Figures Test was exclusively associated with the negative dimension of schizotypy. The implications, therefore, of these results for putative visuo-spatial deficits underlying negative schizotypy and negative psychotic symptoms can serve to motivate further research on more specific cognitive and perceptual mechanisms that are impaired in, and might be responsible for, the negative symptomatology in schizophrenia. 


\section{References}

Ananth, J., Burgoyne, K. S, Gadasalli, R., \& Aquino, S. (2001). How do the atypical antipsychotics work? Journal of Psychiatry and Neuroscience, 26(5), 385-394.

Baruch, I., Hemsley, D. R., \& Gray, J. A. (1988). Differential performance of acute and chronic schizophrenics in latent inhibition task. Journal of Nervous and Mental Disease, 176, 598-606.

Bentall, P. R., Claridge, G. S., \& Slade, P. D. (1989). The multidimensional nature of schizotypal traits: a factor analytic study with normal subjects. British Journal of clinical psychology, 28, 363-375.

Bilder, R. M., Lipschutz-Broch, L., Reiter, G., \& Geisler, S. H. (1992). Intellectual deficits in first episode schizophrenia: evidence for progressive deterioration Schizophrenia Bulletin, 18(3), 437-448.

Braff, D. L. (1993). Information processing and attention dysfunction in schizophrenia. Schizophrenia Bulletin, 19(2), 233-259.

Burch, G. S., Steel, C., \& Hemsley, D. R. (1998). Oxford-Liverpool Inventory of Feelings and Experiences: reliability in an experimental population. British Journal of Clinical Psychology, 37(1), 107-108.

Carroll, J. B. (1993). Human cognitive abilities. Cambridge: Cambridge University Press.

Chey, J., \& Holzman, P. S. (1997). Perceptual organization in schizophrenia: utilization of the Gestalt principles. Journal of Abnormal Psychology, 106(4), 530538.

Della Salla, S., Gray, C., Spinnler, H., \& Trivelli, C. (1998). Frontal lobe functioning in man: the riddle revisited. Archives of Clinical Neuropsychology, 13(8), 663-682.

Dickman, S. J. (1996). Adverse (and beneficial) consequences of impulsive behaviour. In S. R. Feldman, (Ed.) The psychology of adversity. Amherst, MA: University of Massachusetts Press.

Dickman, S. J. (1993). Impulsivity and information processing. In W. McCown, M. Shure, \& J. Johnson, The impulsive client: theory research and treatment. Washington, DC: American Psychological Association.

Dickman, S. J. (2000). Impulsivity, arousal and attention. Personality and Individual Differences, 28(3), 563-581. 
Eimon, M. C., Eimon, P. L., \& Cermak, S. A. (1983). Performance of schizophrenic patients on a motor-free visual perception test. American Journal of Occupational Therapy, 37(5), 327-332.

Ekstrom, R. B., French, J. W., Harman, H. H., \& Dermen, D. (1976). Kit of factor referenced cognitive tests. Princeton, N.J.: Educational Testing Service.

Engle, R. W., Tuholski, S. W., Laughlin, J. E., \& Conway, A.R.A. (1999). Working memory, short-term memory, and general fluid intelligence: a latent variable approach. Journal of Experimental Psychology: General, 128, 309-331.

Eysenck, H. J., \& Eysenck, S. B. G. (1975). Manual of the Eysenck personality questionnaire. London: Hodder and Stoughton.

Federico, P. A. (1984). Event-related-potential (ERP) correlates of cognitive styles, abilities and aptitudes. Personality and Individual Differences, 5(5), 575-585.

Gold, S., Arndt, S., Nopoulos, P., O’ Leary, D. S., \& Andreasen, N. C. (1999). Longitudinal study of cognitive function in first-episode and recent-onset schizophrenia. American Journal of Psychiatry, 156(9), 1342-1348.

Goodarzi, M. A., Wykes, T., \& Hemsley, D. R. (2000). Cerebral lateralization of global-local processing in people with schizotypy. Schizophrenia Research, 45(1-2), 115-121.

Gray, N. S, Pilowsky, L. S., Gray, J. A., \& Kerwin, R.W. (1995). Latent inhibition in drug naïve schizophrenic patients: relationship to duration of illness and dopamine D2 binding using SPET. Schizophrenia Research, 17(1), 95-107.

Humphreys, G. W., \& Mueller, H. (2000). A search asymmetry reversed by figureground assignment. Psychological Science, 11(3), 196-201.

Jensterle, J., Mlakar, J., Vodusek, D. B., \& Frith, C. D. (2000). Disorganisation in schizophrenia need not result from a failure to inhibit dominant response tendencies. Cognitive Neuropsychiatry, 5(2), 105-121.

Knight, R. A., Manoach, D. S., \& Hershenson, M. (2000). Perceptual organization in schizophrenia: the processing of symmetrical configurations. Journal of Abnormal Psychology, 109(4), 575-587.

Kyllonen, P. C. (1996). Is working memory capacity Spearman's $g$ ? In I. Dennis, \& P. Tapsfield (Eds.), Human abilities: their nature and measurement. Mawhah, N.J.: Lawrence Erlbaum Associates.

Lahti, A. C., Holcomb, H. H., Medoff, D. R., Weiler, M. A., Tamminga, C. A., \& Carpenter, W. T. (2001). Abnormal patterns of regional cerebral blood flow in schizophrenia with primary negative symptoms during an effortful auditory recognition task. American Journal of Psychiatry, 158(11), 1797-1808. 
Lezak, M. (1995). Neuropsychological assessment (3rd ed.). Oxford : OUP

Liddle, P. (1987). The symptoms of chronic schizophrenia: a re-examination of the positive negative dichotomy. British Journal of Psychiatry, 151, 221-234.

Liddle, P. (1988). Schizophrenic syndromes, cognitive performance and neurological dysfunction. Psychological Medicine, 17(1), 49-57.

Lipp, O. V., \& Vaitl, D. (1992). Latent inhibition in human Pavlovian differential conditioning: effects of additional stimulation after preexposure and relation to schizotypal traits. Personality and Individual Differences, 13, 1003-1012.

Lubow, R. E., Ingberg-Sachs, Y, Zahlstein-Orda, N., \& Gewirtz, J. (1992). Latent inhibition in low and high "psychotic prone" normal subjects. Personality and Individual Differences, 13, 563-572.

Lubow, R. E, Kaplan, O., Abramovich, P., Rudnick, A., \& Laor, N. (2000). Visual search in schizophrenia: latent inhibition and novel pop-out effects. Schizophrenia Research, 45, 145-156.

Mason, O., Claridge, G., \& Jackson, M. (1995). New scales for the assessment of schizotypy. Personality and Individual Differences, 53, 727-730.

McKenna, F. P. (1984). Measures of field independence: cognitive style or cognitive ability? Journal of Personality and Social Psychology, 47, 593-603.

Miyake, A., Witzki, A. H., \& Emerson, M. J. (2001). Field dependence/independence from a working memory perspective: a dual-task investigation of the Hidden Figures Test. Memory, 9(6), 445-457.

Nestor, P. G., \& O’Donell, B. F. (1998). The mind adrift: attentional disregulations in schizophrenia. In R. Parasuraman (Ed.), The attentive brain. Cambridge, MA: MIT Press.

Neville, J. W. (1995). Validating the Rorschach measures of perceptual accuracy. Dissertation Abstracts International Section B: The Sciences and Engineering, 55(9), 4128.

Noppe, L. D. (1985). The relationship of formal thought and cognitive styles to creativity. Journal of Creative Behaviour, 9(2), 88-96.

Raven, J. C., Court, J. H., \& Raven, J. (1977). Manual for Raven's Progressive Matrices. London: H.K. Lewis.

Rawlings, D., \& Goldberg, M. (2001). Correlating a measure of sustained attention with a multidimensional measure of schizotypal traits. Personality and IndividualDifferences, 31(3), 421-431.

Schmajuk, N. A. (2001). Hippocampal dysfunction in schizophrenia. Hippocampus, 11(5), 599-613. 
Schmidt, C. P., \& Stephans, R. (1991). Locus of control and field dependence as factors in students' evaluations of applied music instruction. Perceptual and Motor Skills, 73(1), 131-136.

Schwartz, B. D., \& Evans, W. J. (1999). Neurophysiological mechanisms of attention deficits in schizophrenia. Neuropsychiatry, Neuropsychology and Behavioural Neurology, 12(4), 207-20.

Silverstein, S. M., Bakshi, S., Chapman, R. M., \& Nowlis, G. (1998). Perceptual organisation of configural and nonconfigural visual patterns in schizophrenia: effects of repeated exposure. Cognitive Neuropsychiatry, 3(3), 209-223.

Silverstein, S. M., Matteson, S., \& Knight, R.A. (1996). Reduced top-down influence in auditory perceptual organization in schizophrenia. Journal of Abnormal Psychology, 105(4), 663-667.

Tsakanikos, E., \& Reed, P. (in press). Latent inhibition and psychosis-proneness; visual search as a function of pre-exposure to the target and schizotypy level. Personality and Individual Differences.

Van den Bosch, R.J. (1995). Context and cognition in schizophrenia. In J. A. Den Boer, \& H. G. M. Westenberg (Eds.), Advances in the neurobiology of schizophrenia. New York: John Wiley \& Sons.

Vecera, S. P., \& O’Reilly, R. C. (1998). Figure-ground organization and object recognition processes: an interactive account. Journal of Experimental Psychology: Human Perception and Performance, 24(2), 441-462.

Vollema, M. G., \& Van den Bosch, R. J. (1995). The multidimensionality of schizotypy. Schizophrenia Bulletin, 21(1), 19-31. 


\section{Acknowledgements}

The first author is supported by the state scholarship foundation (IKY) of the Greek government. Thanks are due to Andrea Lafourara, Angela Livaditis, Loredana Minini, Dino Petrides, Nick Sevdalis, and Marriane So for their help with the data collection. 
Table 1

Means and standard deviations of the scales on Oxford-Liverpool Inventory of Feeling and Experiences, and their inter-correlations

\begin{tabular}{|c|c|c|c|c|c|c|}
\hline Schizotypy Scale & $M$ & $S D$ & 1 & 2 & 3 & 4 \\
\hline 'Unusual Experiences’ & 10.42 & 5.1 & - & & & \\
\hline ‘Cognitive Disorganization’ & 12.82 & 5.57 & $0.44^{*}$ & - & & \\
\hline 'Introvertive Anhedonia’ & 5.16 & 3.98 & 0.07 & $0.29 *$ & - & \\
\hline 'Impulsive Nonconformity’ & 9.75 & 3.56 & $0.42 *$ & $0.41 *$ & -0.01 & - \\
\hline
\end{tabular}




\section{Table 2}

O-LIFE scales and scores on Raven's Progressive Matrices as predictor variables for the number of correct responses on the Hidden Figures Test

\begin{tabular}{lcccc}
\hline Predictor Variable & $B$ & SEB & Beta & $t$ \\
\hline Raven's Progressive Matrices & 0.24 & 0.10 & 0.19 & $2.14^{*}$ \\
'Unusual Experiences' & -0.14 & 0.13 & -0.11 & -1.07 \\
'Cognitive Disorganization' & -0.01 & 0.13 & -0.06 & -0.49 \\
'Introvertive Anhedonia' & -0.37 & 0.15 & -0.23 & $-2.42^{* *}$ \\
'Impulsive Nonconformity' & 0.73 & 0.19 & 0.40 & $3.91^{* * *}$ \\
\hline
\end{tabular}

${ }^{*} p<0.05,{ }^{* *} p<0.01, p<0.001 * * *$ (two-tailed) 